\title{
The Growing Epidemic of HPV-Positive Oropharyngeal Carcinoma: A Clinical Review for Primary Care Providers
}

\author{
Kevin A. Moore II, MD, and Vikas Mehta, MD
}

While the rate of head and neck cancer has decreased in recent decades, the prevalence of oropharynx cancer has dramatically increased due to human papillomavirus (HPV)-related oropharyngeal cancer. Three of 4 newly diagnosed oropharyngeal carcinomas are HPV-positive, and by 2020 it is projected that the prevalence of this disease will overtake that of HPV-related cervical cancer. Recognized in recent years as a malignant entity distinct from HPV-negative oropharyngeal carcinoma, HPV-positive oropharyngeal cancer is associated with younger age at diagnosis, oral sexual behavior as a primary risk factor, nonspecific presentation, and improved treatment response compared with HPV-negative disease. Early recognition and referral for definitive treatment are paramount in decreasing morbidity and mortality, as well as improving the quality of life of these patients. Primary care providers are in an ideal position to improve patient outcomes through early recognition and referral, as well as coordination of comprehensive care of patients with this potentially devastating disease. Awareness of risk factors, a high index of suspicion, counseling patients and parents on the importance of vaccination against HPV, and coordinated care between primary care providers and specialists are vital to achieving improved outcomes for patients with this increasingly prevalent cancer. (J Am Board Fam Med 2015;28: 498-503.)

Keywords: Cancer, Epidemiology, Head and Neck Cancer, Human Papillomavirus, Oropharyngeal Cancer, Otolaryngology

Oropharynx cancer has been increasing at an epidemic rate over the past several decades, with a prevalence in the United States that has increased $225 \%$ from 1988 to $2004 .{ }^{1}$ This meteoric rise is exclusively a result of human papillomavirus (HPV)related oropharyngeal squamous cell carcinoma (OPSCC). According to recent data, 3 of 4 newly diagnosed oropharyngeal carcinomas are HPVpositive $(\mathrm{HPV}+)$; if current trends continue,

This article was externally peer reviewed.

Submitted 30 October 2014; revised 28 February 2015; accepted 9 March 2015.

From the Louisiana State University School of Medicine, Shreveport (KAM); and the Department of Otolaryngology/ Head and Neck Surgery, Louisiana State University School of Medicine, Shreveport (VK).

Funding: none.

Conflict of interest: none declared.

Corresponding author: Vikas Mehta, MD, Department of Otolaryngology, LSU Health Shreveport, Room 9-203, 1501 Kings Highway, Shreveport, LA 71103 (E-mail: vmeht2@1suhsc.edu).
$\mathrm{HPV}+$ OPSCC prevalence is expected to overtake that of cervical cancer by the year 2020., ${ }^{2,3} \mathrm{HPV}+$ OPSCC has been recognized as a malignant process distinct from HPV-negative (HPV-) OPSCC, with its own epidemiology, tumor biology, presentation, response to treatment, and prognosis.

Because of its rapidly increasing prevalence and often nonspecific presentation, timely diagnosis and treatment of HPV + OPSCC requires a high index of suspicion from primary care providers, who are commonly the first point of professional contact for patients with symptoms of HPV + OPSCC. Awareness of the epidemiology, risk factors, and presentation of these tumors is crucial to producing improved outcomes through rapid referral for diagnosis and definitive treatment of these tumors, as well as maintaining the patient's quality of life. The objective of this article is to increase awareness of $\mathrm{HPV}+$ OPSCC among primary care providers and emphasize the importance of early detection of 
these tumors in decreasing morbidity and mortality for these patients.

\section{Why Does Tumor HPV Status Matter?}

The HPV status of OPSCC is of vital importance because HPV+ OPSCC displays epidemiologic and biological behavior distinct from that of HPV - OPSCC. These differences in behavior affect every aspect of the disease, from initial symptoms noticed by the patient, to tumor location, size, likelihood and timing of lymph node spread, to response to therapy and quality of life after treatment. ${ }^{4} \mathrm{HPV}+$ oropharyngeal cancer is associated with younger age at diagnosis, oral sexual behavior as a primary risk factor, nonspecific presentation, and improved treatment response compared with $\mathrm{HPV}$ - disease. According to a meta-analysis by O'Rorke et al, ${ }^{5}$ patients with HPV + OPSCC have more favorable overall survival (hazard ratio [HR], $0.47 ; 95 \%$ confidence interval [CI], 0.35-0.62); disease-specific survival (HR, 0.26; 95\% CI, 0.170.39 ); and disease-free survival (HR, 0.37; $95 \% \mathrm{CI}$, $0.22-0.63)$. A prospective trial evaluating survival and therapeutic response based on tumor HPV status found that the estimated overall survival rate for patients with HPV+ OPSCC was 95\% (95\% CI, $87-100 \%)$ at 2 years. By contrast, estimated overall survival for patients with HPV- OPSCC was only $62 \%(95 \% \mathrm{CI}, 49-74 \%)$ at 2 years. ${ }^{6}$ It is increasingly being recognized that because these tumors behave differently, they can be treated differently as well. In fact, deescalation of treatment for these tumors is currently being investigated in an attempt to provide more appropriate therapeutic interventions resulting in decreased morbidity and mortality and improved quality of life. ${ }^{7}$

\section{Epidemiology of HPV + OPSCC}

While the overall prevalence of head and neck cancer has been decreasing, the prevalence of $\mathrm{HPV}+$ OPSCC has increased dramatically in recent years. Before the year 2000, HPV+ OPSCC represented $40.5 \%$ of all OPSCC cases, with the remainder being the HPV - variety associated with the classic head and neck cancer risk factors of smoking and alcohol use. ${ }^{2}$ Since $2005,>70 \%$ of OPSCC cases have been $\mathrm{HPV}+$, representing a $70 \%$ increase in the proportion of HPV + disease. $^{2}$ Increased awareness of the carcinogenic role of cigarette smoking, as well as the increasing preva- lence of high-risk HPV related to sexual behaviors, are thought to have contributed to this shift. In an analysis of 116 cases of HPV+ OPSCC, 87.9\% were specifically associated with the HPV-16 serotype. ${ }^{3}$ In a cross-sectional study by Gillison et $\mathrm{al},{ }^{8}$ prevalence of oral infection in the general population with any high-risk HPV type was 3.7\% (95\% CI, 3-4.6), and prevalence of HPV-16 specifically was $1 \%(95 \% \mathrm{CI}, 0.7-1.3)$. The prevalence of oral high-risk HPV infection underscores the risk for increasing prevalence of HPV + OPSCC in the future.

Patients with these tumors may be quite different from patients in whom HPV- OPSCC would be suspected. These tumors tend to affect younger patients who are more likely to be college-educated and white, and who may not have many of the "classic" squamous cell carcinoma risk factors such as heavy tobacco and alcohol use. Gillison et $\mathrm{al}^{9}$ found that the median age of patients with HPV+ tumors was 54 years, versus 58 years for HPVtumors $(P=.002)$, and they were more likely to be college-educated $(P=.003)$, be white $(P=.006)$, and have an annual income of $\geq \$ 50,000(P<.001)$. Strong risk factors for $\mathrm{HPV}+$ disease include a history of multiple vaginal or oral sexual partners and marijuana use (odds ratio [OR], 4.5; 95\% CI, 1.6-13). The ORs for HPV+ OPSCC increased with both the intensity $\left(P_{\text {trend }}=.003\right)$ and duration $\left(P_{\text {trend }}=.005\right)$ of marijuana use. Nontobacco smokers who had $\geq 5$ joint-years of marijuana use had an OR of 11 (95\% CI, 1.6-74) compared with sporadic users or nonusers of marijuana.

Perhaps the most important risk factor is oral sexual behavior. The odds of HPV+ OPSCC increase significantly with the lifetime number of oral sex partners $\left(P_{\text {trend }}=0.011\right)$, with a univariate OR of 5.7 (95\% CI, 1.9-13.7) for individuals with 6 to 15 lifetime oral sex partners. ${ }^{9}$ In fact, D'Souza et $\mathrm{al}^{10}$ found that the age- and race-related differences in the prevalence of oral HPV-16 infection-and likely HPV+ OPSCC, since HPV-16 is responsible for the vast majority of cases - can be explained based on differences in oral sexual behaviors. They found that neither white race nor younger age were independently associated with oral HPV-16 once they adjusted for oral sexual behavior. ${ }^{10}$ Younger populations are more likely to engage in oral sex, and this seems to be the driving force for the epidemic increase that has occurred. 
Although patients with HPV+ OPSCC are less likely to be chronic smokers, smoking while exposed to HPV increases the likelihood of retention of HPV in the oropharynx (through its local immune-suppressing effects) and thus developing HPV related oropharyngeal cancer later in life. Patients with HPV often have a history of smoking as adolescents or in their early 20s. In addition, smoking exposure at diagnosis and during treatment has been shown to be a significant factor in response to therapy as well as survival. In an analysis of a large trial of patients with oropharynx cancer treated with either radiation or chemoradiation, patients with HPV+ OPSCC were more likely to have never smoked $(21.3 \%$ vs $5.2 \% ; P<$ $.001)$, were less likely to be current smokers $(37.3 \%$ vs $67.8 \% ; P<.001)$, and had less exposure to smoking overall (median, 29 vs 45.9 pack-years; $P=.02$ ) compared with patients with HPVOPSCC. ${ }^{11}$ In addition, smoking exposure at diagnosis was found to increase mortality by $1 \%$ per pack-year, even for patients with HPV+ OPSCC, and the hazard of death was significantly increased for patients who smoked during therapy (HR, 2.19; 95\% CI, 1.46-3.28). ${ }^{11}$ Currently, no other major carcinogens have been identified as significant risk factors for HPV+ OPSCC. The risk factor profile for these patients is strikingly different from that of patients with "traditional" head and neck cancer and is driven primarily by differences in oral sexual behaviors.

\section{Presentation and Characteristics of HPV+ OPSCC}

In addition to affecting patients with different risk factors, as described above, HPV+ OPSCC tumors display clinical features and a treatment response unlike that of HPV- OPSCC. HPV+ OPSCC most commonly arises from the lymphoid tissues of the palatine tonsils or base of tongue, which are characterized by a disrupted basal cell layer that functions to enhance the immunologic role of this tissue. It has been postulated that this histology contributes to the propensity of these tumors to metastasize earlier, whereas primary tumors are smaller and even occasionally present as cervical metastases with unknown primaries. ${ }^{12}$

McIlwain et $\mathrm{al}^{4}$ demonstrated that the most common initial symptom of HPV + OPSCC is a neck mass (51\% of patients), whereas only $18 \%$ of patients with HPV - OPSCC presented with this symptom. Although HPV+ OPSCC has a high rate of cervical metastases, even when the primary tumor is small and asymptomatic, it does not usually present with distant metastasis. For HPVOPSCC, sore throat was the most common initial symptom (53\% of patients). Other symptoms included dysphagia, visualized mass, globus sensation, and otalgia, among others ${ }^{4}$ (Table 1). Although many of these symptoms are also characteristic of more common benign disease processes, persistent symptoms or a lack of response to standard treatment warrant further investigation with minimal delay.

\section{Evaluation and Diagnosis}

Evaluation of these patients should (as always) begin with a thorough history and physical examination of the head and neck, with special attention paid to the common symptoms listed above. A review of symptoms at each visit ideally includes those symptoms as well, since many patients may not bring up the symptoms initially because of the belief that they are minor or will correct themselves. ${ }^{13}$ Physical examination should include close inspection of the tonsillar complex, inspection and palpation of the base of tongue, and a careful examination for cervical lymphadenopathy. Because currently there are no standard tests to evaluate for precancerous OPSCC lesions (such as the Papanicolaou test and HPV DNA testing for cervical cancer), a detailed review of systems and physical examination remain the most important method for early detection.

Table 1. Most Common Presenting Symptoms of Human Papillomavirus-Positive Oropharyngeal Cancer from a retrospective study of 430 patients by McIlwain et al. ${ }^{4}$

\begin{tabular}{lc}
\hline Presenting Symptoms & Prevalence (\%) \\
\hline Neck mass & 51 \\
Sore throat & 33 \\
Dysphagia & 16 \\
Visualized mass & 13 \\
Globus sensation & 10 \\
Odynophagia & 9 \\
Otalgia & 7 \\
\hline
\end{tabular}

Data obtained from McIlwain et al. ${ }^{4}$ 
Suspicious lesions found during the physical examination require timely diagnostic workup and referral to an otolaryngologist. As mentioned above, the most common physical examination presentation for patients with HPV+ OPSCC is a unilateral neck mass because of high rate of cervical lymph node metastases, even when the primary tumor volume is small. In the case of an adult with a unilateral neck mass, fine-needle aspiration (FNA) and computed tomography (CT; Figure 1) are 2 diagnostic modalities that provide useful information and can be performed in the primary care setting, if available. FNA is associated with minimal discomfort to the patient and can be performed in the office, although it is dependent on the experience of the physician performing the procedure and the availability of cytologic examination. ${ }^{14}$ In one study the sensitivity and specificity for detecting head and neck cancer via FNA of lymph node metastases were $88.2 \%$ and $100 \%$, respectively. Cells obtained by FNA can also be examined for HPV biomarkers, further increasing their diagnostic value. ${ }^{14} \mathrm{CT}$ can provide information related to primary tumor size and location as well as metastatic spread ${ }^{15}$ (Figure 1). A preliminary workup of suspected OPSCC should always include a CT scan of the head and neck. Any

Figure 1. Computed tomography scan of a 44-year-old male who presented with a unilateral neck mass. The arrow indicates location of the mass.

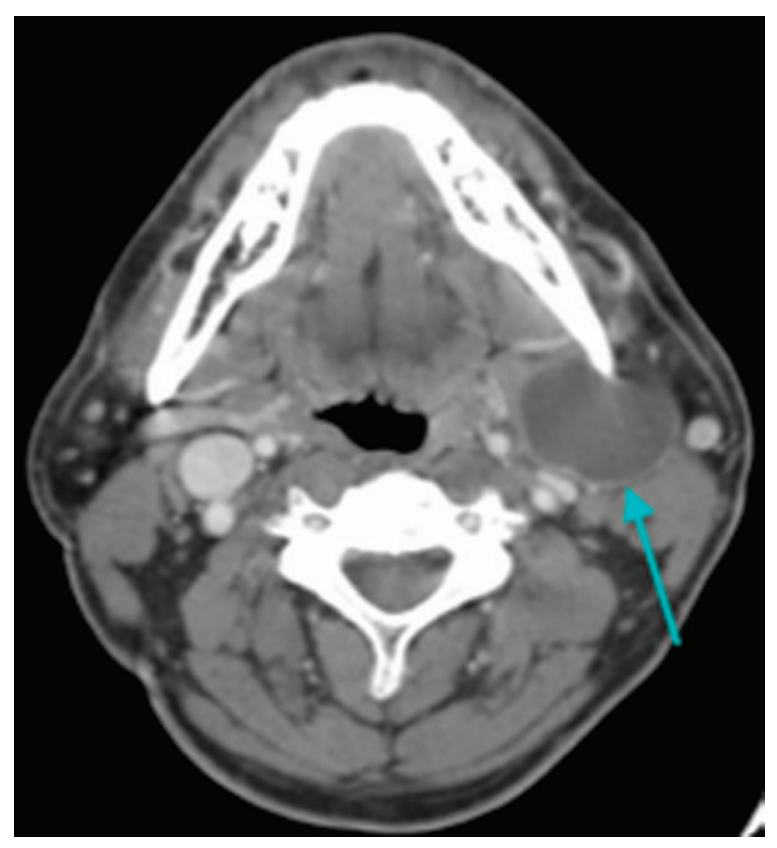

patient who presents with a unilateral neck mass and who does not respond to a short course of antibiotics or has a history that is suspicious for head and neck cancer should receive an FNA and a CT scan in a timely manner. As noted above, patients with a unilateral neck mass that have HPV + OPSCC will become increasingly common in the future, thus making a high index of suspicion crucial for early identification and referral. Further diagnostic evaluation, such as positron emission tomography/CT and surgical biopsies, if necessary, can be obtained after referral to an otolaryngologist/head and neck oncologist.

\section{Role of the Primary Care Provider}

When dealing with a cancer that can have such nonspecific initial symptoms yet such a profound impact on quality of life, the role of the primary care provider is critically important. Awareness of HPV + OPSCC and early referral for diagnosis are crucial to increasing the rate of successful treatment and minimizing morbidity from disease and treatment. While chemoradiation and surgical modalities are combined for treatment of later stages of the disease, earlier stages can be treated with surgical excision or radiation alone in some cases. ${ }^{16}$ More conservative treatment of earlier stages of the disease decreases potential morbidity from xerostomia or dysphagia, which can be associated with radiation therapy or side effects from chemotherapeutic regimens. Surgical excision of smaller lesions can also result in the decreased likelihood of functional impairment related to resection of the mass.

Quality of life can also be improved through monitoring the patient's nutritional status before, during, and after treatment. Patients with OPSCC are at an increased risk for weight loss resulting from symptoms such as dysphagia or odynophagia, as well as to treatment-related complications such as functional impairment from surgery or oral mucositis, nausea, and vomiting associated with chemotherapy. As stated above, cigarette smoking can adversely affect treatment response and outcomes for these patients, so counseling for smoking cessation is important before, during, and after treatment. Coordination of care between the primary care provider, head and neck oncologist, and speech/language pathologist can minimize the impact of these factors on the patient's quality of life. ${ }^{17}$ 
Achieving a successful outcome for these patients, which involves a combination of early recognition, prompt therapy, and improved quality of life, relies heavily on heightened awareness in addition to superior coordination of care by skilled primary care providers.

\section{Vaccination}

The sharply increasing prevalence of $\mathrm{HPV}+$ OPSCC further establishes the importance of vaccination against HPV in all eligible patients, both male and female. Two vaccines against HPV are currently available: one that targets HPV-16 and -18 (Cervarix; GlaxoSmithKline) and one that targets HPV-6, -11, -16, and -18 (Gardasil; Merck Sharp \& Dohme Corp.). HPV+ OPSCC is most often caused by HPV-16 and infrequently by HPV18 ; thus both vaccines are effective in preventing oral transmission of the cancer-causing virus. HPV-6 and - 11 are most often implicated in benign warts in the anogenital area. While the specific effect of HPV vaccination on HPV + OPSCC itself has not been evaluated yet, the currently available HPV-16/-18 vaccine has recently been found to be even more efficacious against oral HPV infection (vaccine efficacy, 93.3\%; 95\% CI, 62.5$99.7 \%$ ) than against cervical HPV infection (vaccine efficacy, $72.0 \%$; 95\% CI, $63.0-79.1 \%$ ) in a randomized trial of young women in Costa Rica. ${ }^{18}$ This efficacy is likely to also be observed in men. In a 2008 analysis of the cost-effectiveness of routine $\mathrm{HPV}$ vaccination of 12 -year-old girls, the cost per quality-adjusted life-year was $\$ 3,906$ when cervical, anal, vaginal, vulvar, and oropharyngeal cancers, as well as herd immunity effects, were considered. ${ }^{19}$ As a comparison, in a 2006 study the cost per quality-adjusted life-year for vaccinating adults aged 50 to 64 years against influenza was $\$ 28,044 .^{20}$ Vaccination against high-risk HPV types is crucial in preventing these devastating cancers in the future. The HPV vaccine is recommended for children and adolescents aged 9 to 26 years, and parents should be counseled with regard to its cancerpreventing potential. The current cost is approximately $\$ 360$, which is covered through most public and private insurances.

\section{Conclusion}

As is true with many other disease processes, primary care providers are in an optimal position to ensure the highest level of care for patients with $\mathrm{HPV}+\mathrm{OPSCC}$. Awareness of and counseling on risk factors, early diagnosis, referral for treatment, and close monitoring for nutritional status and other quality of life issues can have an extraordinary effect on the care of these patients. Although the prevalence of this disease has increased sharply in recent years, analysis of outcomes in these patients has fortunately shown that they tend to have markedly improved outcomes with regard to treatment failure and overall survival compared with patients with HPV- OPSCC. ${ }^{5}$ The typical picture of the patients with oropharyngeal cancer is shifting, but increased awareness of these changes, emphasis on vaccination against $\mathrm{HPV}$, and prompt, coordinated care between primary care providers and specialists can continue to decrease morbidity and mortality from this otherwise devastating disease.

The authors thank Talicia Tarver for her assistance with manuscript preparation.

\section{References}

1. Pytynia KB, Dahlstrom KR, Sturgis EM. Epidemiology of HPV-associated oropharygeal cancer. Oral Oncol 2014;50:380-6.

2. Mehanna H, Beech T, Nicholson T, et al. Prevalence of human papillomavirus in oropharyngeal and nonoropharyngeal head and neck cancer-systematic review and meta-analysis of trends by time and region. Head Neck 2013;35:747-55.

3. Chaturvedi AK, Engels EA, Pfeiffer RM, et al. Human papillomavirus and rising oropharyngeal cancer incidence in the United States. J Clin Oncol 2011; 29:4294-301.

4. McIlwain WR, Sood AJ, Nguyen SA, Day TA. Initial symptoms in patients with HPV-positive and HPVnegative oropharyngeal cancer. JAMA Otolaryngol Head Neck Surg 2014;140:441-7.

5. O'Rorke MA, Ellison MV, Murray LJ, Moran M, James J, Anderson LA. Human papillomavirus related head and neck cancer survival: a systematic review and meta-analysis. Oral Oncol 2012;48:1191201.

6. Fakhry C, Westra WH, Li S, et al. Improved survival of patients with human papillomavirus-positive head and neck squamous cell carcinoma in a prospective clinical trial. J Natl Cancer Inst 2008;100:261-9.

7. Zaravinos A. An updated overview of HPV-associated head and neck carcinomas. Oncotarget 2014;5: 3956-69.

8. Gillison ML, Broutian T, Pickard RKL, et al. Prevalence of oral HPV infection in the United States, 2009-2010. JAMA 2012;307:693-703. 
9. Gillison ML, D'Souza G, Westra W, et al. Distinct risk factor profiles for human papillomavirus type 16-positive and human papillomavirus type 16-negative head and neck cancers. J Natl Cancer Inst 2008;100:407-20.

10. D'Souza G, Cullen K, Bowie J, Thorpe R, Fakhry C. Differences in oral sexual behaviors by gender, age, and race explain observed differences in prevalence of oral human papillomavirus infection. PLoS One 2014;9:e86023.

11. Gillison ML, Zhang Q, Jordan R, et al. Tobacco smoking and increased risk of death and progression for patients with p16-positive and p16-negative oropharyngeal cancer. J Clinl Oncol 2012;30: 2102-11.

12. Howard JD, Chung CH. Biology of human papillomavirus-related oropharyngeal cancer. Semin Radiat Oncol 2012;22:187-93.

13. Rogers SN, Vedpathak SV, Lowe D. Reasons for delayed presentation in oral and oropharyngeal cancer: the patients perspective. Br J Oral Maxillofac Surg 2011;49:349-53.

14. Jakscha et al. The clinical impact of p16 status in fine-needle aspirates of cervical lymph node metas- tasis of head and neck squamous cell carcinomas. Eur Arch Otorhinolaryngol 2013;270:661-7.

15. Corey AS, Hudgins PA. Radiographic imaging of human papillomavirus related carcinomas of the oropharynx. Head Neck Pathol 2012;6:S25-40.

16. Broglie MA, Soltermann A, Haile SR, et al. Quality of life of oropharyngeal cancer patients with respect to treatment strategy and p16 positivity. Laryngoscope 2013;123:164-70.

17. Pfister DG, Ang KK, Brizel DM, et al; National Comprehensive Cancer Network. Head and neck cancers, version 2.2013. Featured updates to the NCCN guidelines. J Natl Compr Canc Netw 2013;11:917-23.

18. Herrero R, Quint W, Hildesheim A. Reduced prevalence of oral human papillomavirus 4 years after bivalent hpv vaccination in a randomized clinical trial in Costa Rica. PLoS One 2013;8:e68329.

19. Chesson HW, Ekwueme DU, Saraiya M, Markowitz LE. Cost-effectiveness of Human Papillomavirus Vaccination in the United States. Emerging Infectious Diseases 2008; 14:244-251.

20. Maciosek MV, Solberg LI, Coffield AB, Edwards NM, Goodman MJ. Influenza vaccination health impact and cost effectiveness among adults aged 50 to 64 and 65 and older. Am J Prev Med 2006;31:72-9. 